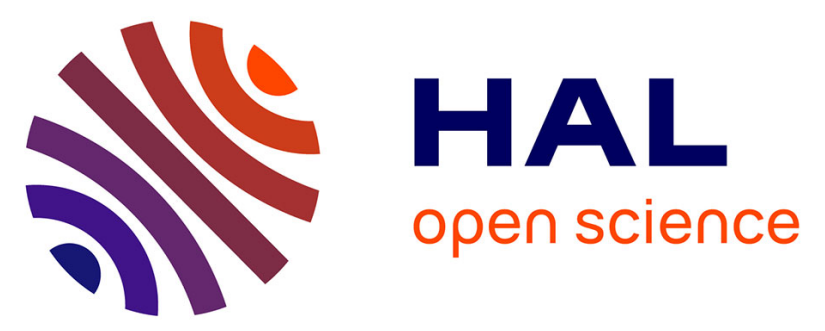

\title{
The light is red: Uncertainty behaviours displayed by pedestrians during illegal road crossing
}

Mathilde Jay, Anne Régnier, Anaïs Dasnon, Killian Brunet, Marie Pelé

\section{To cite this version:}

Mathilde Jay, Anne Régnier, Anaïs Dasnon, Killian Brunet, Marie Pelé. The light is red: Uncertainty behaviours displayed by pedestrians during illegal road crossing. Accident Analysis \& Prevention, 2020, 135, pp.105369. 10.1016/j.aap.2019.105369 . hal-02502858

\section{HAL Id: hal-02502858 \\ https://hal.science/hal-02502858}

Submitted on 21 Dec 2021

HAL is a multi-disciplinary open access archive for the deposit and dissemination of scientific research documents, whether they are published or not. The documents may come from teaching and research institutions in France or abroad, or from public or private research centers.
L'archive ouverte pluridisciplinaire HAL, est destinée au dépôt et à la diffusion de documents scientifiques de niveau recherche, publiés ou non, émanant des établissements d'enseignement et de recherche français ou étrangers, des laboratoires publics ou privés.

\section{다)(1) $(5$}

Distributed under a Creative Commons Attribution - NonCommerciall 4.0 International 
1 The light is red: uncertainty behaviours displayed by pedestrians during illegal road 2 crossing

3

4 Mathilde Jay $^{1}$, Anne Régnier $^{1}$, Anaïs Dasnon ${ }^{1}$, Killian Brunet ${ }^{1}$, Marie Pelé ${ }^{1}$

5

$6 \quad{ }^{1}$ Université de Strasbourg, CNRS, IPHC UMR 7178, F-67000 Strasbourg, France

7

8 Corresponding author:

9 Marie Pelé

10 marie.pele@iphc.cnrs.fr

11 23, rue Becquerel -67087 Strasbourg, FRANCE

12

13

14

15

16

17

18

19

20

21

22

23

24

25

26

27

28

29

30

31

32 


\section{ABSTRACT}

Road accidents involving pedestrians are a reality of urban life. Pedestrian risk is now well known and documented from the perspective of drivers. However, pedestrian behaviour plays a central role in road accidents, notably in terms of illegal road crossing at signalized intersections. This study focuses on pedestrians crossing illegally at a signal light, and specifically investigates uncertainty behaviour, also referred to as hesitation, which occurs when a pedestrian slows down or stops his/her crossing movement then (1) abandons the crossing by returning to the kerb or (2) accelerates to cross the road more quickly. We sought to understand the causes of this behaviour in France and Japan, two countries where interesting differences have already been demonstrated in the way pedestrians behave. The results show a longer period of uncertainty for pedestrians in Japan compared to France. Japanese pedestrians also hesitated longer when they were alone. This study demonstrates a tendency to speed up if there are a number of pedestrians already crossing the road, but abandoning behaviours were more frequently observed than acceleration. This study confirms that pedestrians may misevaluate the moment to cross and hesitate when they realise that they have made a mistake, thus increasing the risk of an accident. These results could help to find solutions that prevent illegal and dangerous road-crossing behaviours.

KEYWORDS: illegal road-crossing; pedestrian; uncertainty; hesitation; decision-making; culture

(1)

2

63




\section{INTRODUCTION}

In 1896, Bridget Driscoll was killed by a motor vehicle during a demonstration in the United Kingdom; her death is the first recorded case of pedestrian road fatality (Porter, 1998). The number of road traffic accidents has since continued to rise, with pedestrians representing $22 \%$ of world road traffic death (World Health Organization, 2015, WHO/NMH/NVI/15.6). Despite the efforts made by governments to enhance road safety practices, there are still too many accidents involving pedestrians, most of which occur in urban agglomerations. In France, collisions with pedestrians represent $14 \%$ of lethal road accidents, and $67 \%$ of these deaths occur in the urban environment (ONISR, French Observatory of Road Safety, 2017). These deaths are all the more inacceptable given their largely predictable and preventable nature. Indeed, risks to pedestrians are now well known and documented in terms of driver behaviour (for speeding, see the review by Rosén et al., 2011; for drink driving see Hingson and Winter, 2003) to road infrastructures (see Bunn et al., 2003; Hamed, 2001) and vehicle design (see Desapriya et al., 2010).

Another key factor of pedestrian safety is the behaviour of the pedestrians. Zhuang and $\mathrm{Wu}(2011)$ showed that $66 \%$ of pedestrians crossing outside signalized intersections in China did not check for oncoming vehicles before crossing, and $16.1 \%$ did not even do so whilst crossing. Among pedestrians who saw an approaching vehicle after stepping off the kerb, 40.6\% stopped crossing, $11.4 \%$ stepped backwards and $31.9 \%$ accelerated to complete the crossing. In this example, more than $40 \%$ of pedestrians demonstrated uncertainty behaviours. The authors observed many instances where pedestrians were using cell phones or listening to music while walking or even while crossing roads. The risky pedestrian behaviour described in this case is also problematic in the context of illegal road-crossings at signalized intersections (Diaz, 2002; Preusser et al., 2002; Evans and Norman, 1998; Evans and Norman, 2003; Rosenbloom, 2009; King et al., 2009; Xu et al., 2013).

Pedestrian behaviours vary according to the socio-demographic traits of individuals. Men show more risky behaviours than women, who display more compliance and conformity (Osman, 1982; Granié, 2011). These behaviours can also vary according to countries and social conventions (Sueur et al., 2013). For instance, French pedestrians crossed against the red light much more often than their Japanese counterparts (41.9\% vs 2.1\%) (Pelé et al., 2017). As unsafe behaviours, pedestrian infractions can be classified as either errors or violations (Reason, 1990). While an error is unintentional and associated with individual information, violation is deliberate and associated with social factors (Reason, 1990). Social information (gained from surrounding people) is an important factor in collective human 
phenomena and followership (Faria et al., 2010; Boos et al., 2014). For instance, Dyer and colleagues (2009) showed that a small percentage of informed individuals (5\%) was sufficient to guide a larger group of uninformed people to a target place. At a signalised intersection, some pedestrians do not check the light colour but simply follow when other pedestrians have already started to cross (Pelé et al., 2019). Trusting wrong or unreliable information and following someone who is crossing without first checking the light colour (personal information) leads to an increased risk of accidents and injuries (Rosenbloom, 2009). Indeed, a conflict between these two types of information (social vs personal) may lead the pedestrian to change his/her behaviour while already crossing. Our study focuses on this specific behavioural change in pedestrians crossing illegally at a signal light. We have called this uncertainty behaviour (also referred to as hesitation). We define this specific behaviour as the moment the pedestrian slows down or stops his/her crossing movement then either (1) abandons the crossing by turning back to the kerb or (2) accelerates to cross more quickly. Both of these reactions are potentially dangerous for the pedestrian, who may be hit by vehicles driving on inner or outer road lanes.

To our knowledge, this is the first study to investigate the factors (sociodemographic and environmental) affecting uncertainty behaviour. This specific behaviour is a clear signal that the pedestrian has misevaluated the situation, and it deserves in-depth analysis. We also sought to identify any factors that could affect the abandon and acceleration rates observed in pedestrians. This was achieved by studying the behaviours of pedestrians crossing streets in two different countries: Japan and France. We have already demonstrated interesting differences in the way pedestrians behave in these two countries (Sueur et al., 2013; Pelé et al., 2017). First, there was a higher frequency of infractions in France than in Japan. In view of this finding, we predict a greater relative number of uncertainty behaviours in French pedestrians. Women and the elderly are expected to show more uncertainty behaviours and the longest uncertainty times in comparison to other categories of pedestrians when crossing at the red light. Indeed, women appear to focus more on the behaviour of other pedestrians at the time of crossing (i.e. prioritizing social information) (Tom and Granié, 2011) while older pedestrians (> 65 years old) appear to prioritize their course of travel over other environmental information (Tapiro et al., 2016a). Men and the youngest pedestrians are expected to accelerate after hesitation. At a pedestrian crossing red light, combining the number of pedestrians already crossing with the pedestrian departure order (rank) should increase the rate of illegal crossings and subsequently the rate of uncertainty behaviours, as pedestrians show mimetic process and trust in social information (Faria et al., 2010; Bode et 
al., 2015). We also expect any pedestrians who are using their phones or accompanied by another person to show more uncertainty behaviours than other pedestrians, as these factors are known as distractors (Neider et al., 2010; Tapiro et al., 2016b; Tapiro et al., 2018). Moreover, the probability of abandoning instead of accelerating should increase with the number of lanes on the road, as the risk and the perception of risk increase with the crossing distance.

\section{MATERIALS \& METHODS}

\section{Study sites}

Pedestrians were observed at three sites in Strasbourg, France and at four sites in Nagoya, Japan (see details in Table 1). On each site, the speed of vehicles was limited to $50 \mathrm{~km} / \mathrm{h}$. The speed of pedestrians when crossing the road was estimated by scoring the speed of 20 random pedestrians at each site. It was not significantly different from one site to another (permutation test for independent samples: $\operatorname{maxT}=2.22, \mathrm{p}=0.168$ ). At some sites, vehicles approaching the intersections were allowed to enter the road despite the green light for pedestrians; these drivers were aware that crossing pedestrians had priority and drove much more slowly than vehicles that were heading straight on. However, the driver of an approaching vehicle may be less careful if pedestrians cross at a red light, as the former has the right of way. Pedestrians therefore run a much higher risk of being injured when crossing at a red light. There was no button for pedestrians to trigger the green light at any of the sites.

\section{Data scores}

Data were scored over a six-day period for each site, for one hour per day during working days and between 12:00 and 17:00 to ensure that data included different groups of pedestrians (workers, families with children, retirees, etc.) but also excluded movements generated by social events (tourism, festivals, concerts, etc.). Observation time then varied within locations to get a wider pool of pedestrians. This scoring duration is sufficient to provide a large dataset (Faria et al., 2010; Sueur et al., 2013; Yannis et al., 2013). A video camera was set up in order to score the light colour in locations that were chosen to ensure that crossing pedestrians were visible at all times. We used the behavioural sampling method (Altmann, 1974) to score the behaviours of pedestrians in one direction only, i.e. those recorded by the camera. Pedestrians were not informed about the purpose of the study to avoid any modification of their behaviour. As both cities are touristic, pedestrians are accustomed to seeing tourists taking pictures or videos. Our camera was placed on the other 
side of the road to the starting point of pedestrians. It was not placed directly in front of the crosswalk but was located slightly to one side to make it less of a distraction for pedestrians before and during crossing. None of the pedestrians who noticed the camera changed their direction. To avoid influencing pedestrian behaviour, we purposely did not take any other equipment such as counters or pocket PCs. Any observations of road-crossing that were hampered by a visual obstacle (i.e. a car or a truck in front of the video camera) and the observations occurring immediately before and after it were removed from the analyses. We also removed data in which cyclists and tourists were among the pedestrians. We differentiated tourists from citizens without difficulty, as they were mostly in large groups accompanied by a guide, or carried specific equipment (camera or travel guide) and could be dressed differently to local citizens.

\section{Research ethics}

This study only involves anonymous observations and anonymous data scores. We followed the ethical guidelines for research of both countries and received ethical approval from both the Institut Pluridisciplinaire Hubert Curien, Strasbourg, France and the Primate Research Institute, Kyoto University, Japan to conduct our observations. As all data were anonymous, focal individuals were given sequential numerical identities according to the day of observation, the crossing concerned and the time of the road crossing. To avoid influencing pedestrians, the experimenters did not approach them. However, pedestrians had the opportunity to learn about the study by an informative medium in their language (French or Japanese) if requested after seeing the camera. If they wished to do so, they also had the possibility to obtain an email address and phone number to contact the corresponding author at a later date. Neither of the two Japanese pedestrians nor the three French ones who asked for information refused to participate in the study.

\section{Data analyses}

Uncertainty behaviours were analysed from video recordings by two teams of two coders (AR with $\mathrm{AD}$ and $\mathrm{MJ}$ with $\mathrm{KB}$, each team coding for French and Japanese videos), using BORIS software (Friard and Gamba, 2016) and demonstrating all coder reliability of $80 \%$. We only scored and analysed uncertainty behaviours observed at the red light on pedestrian crossings. However, in order to have a rate of uncertainty behaviour per illegal road-crossings, we also scored the number of illegal road-crossings behaviours in France and in Japan, regardless of the occurrence of uncertainty behaviour. 
We defined the start of a crossing as the moment the pedestrian lifted one of his/her feet to cross. The uncertainty behaviour was defined as the moment the pedestrian stopped his/her crossing movement, or slowed down. This hesitation was then followed by one of two possible behaviours, i.e. scenario 1 , the abandon of the road crossing, which is characterized by returning to the kerb, or scenario 2, acceleration, characterized by an acceleration of the crossing movement (Figure 1). These two possible behaviours following hesitation will hereafter be referred to as "subsequent behaviours". We never observed a pedestrian who continued to cross at the same speed after hesitating, with speed estimated according to the number of steps per second.

Using these behavioural sequences, we defined uncertainty time as the time comprised between the beginning of the uncertainty behaviour and the beginning of the following behaviour (abandon or acceleration).

To determine the environmental and sociodemographic factors affecting uncertainty behaviour and its possible consecutive behaviours, we scored the following variables for each focal pedestrian: - gender (male or female), - age, estimated at 10-year intervals from 0-9, 10-19 [ . . ] to 70-89.

- country (France or Japan),

- the number of lanes to be crossed,

- whether or not the focal pedestrian was using their phone,

- whether or not the pedestrian was accompanied (i.e. the pedestrian was talking with another person or walking very closely and synchronously with her/him, arriving at the road and crossing it at the same time),

- the total number of pedestrians involved in the road-crossing event, second as 2 , and so on. No uncertainty behaviour was noted after the sixth rank.

\section{Statistical analyses}

We first used chi-square tests to assess differences between countries and between genders. To evaluate the effect of age on uncertainty, we calculated the percentage of uncertainty behaviours (i.e. pedestrians as one behaviour per pedestrian) in each age category. Two samples were used for this purpose: (1) a random sample of 1,278 pedestrians $(\mathrm{N}=551$ for France and N=727 for Japan, with 2x10 minutes scorings for each site on two different videos) and (2) our sample of 118 pedestrians crossing at the red light and showing 
uncertainty in each country ( $\mathrm{N}=80$ for France and $\mathrm{N}=38$ for Japan, see details for each site in Table 1). For each sample, we divided the number of individuals per age category by the total number of uncertainty behaviours $(* 100)$. We then calculated the differences between the two percentages in each age category: negative values indicated that fewer individuals showed uncertainty behaviours than the average for a given age category, while positive values indicated that more individuals showed uncertainty behaviours than the average. A Kolmogorov-Smirnov test was realised to compare the distributions of the hesitation frequency according to departure order. For this test, the mean departure order rank \pm standard deviation per uncertainty behaviours is given per country.

A generalized linear model (GLM) was used to test the influence of the independent variables on our response variables. We first analysed the uncertainty time using a Gamma law (link $=\log$ ) with the following independent variables: the age and the gender of the hesitating pedestrian, whether s/he was using a phone, whether s/he was accompanied by at least one other person, the country, the number of lanes, the number of waiting pedestrians, the order of pedestrian departure, the [country*gender] interaction and the [country*accompanied] interaction. Another possible biological and viable interaction would have been [country*age], but this was not tested due to insufficient data per age category. We then analysed the probability of abandon or acceleration using a Binomial law (abandon noted as 1 , acceleration noted as 0 ) and with the following dependent variables: the age and the gender of the hesitating pedestrian, whether s/he was using a phone, whether s/he was accompanied by at least one other person, the country, the number of lanes, the number of waiting pedestrians and the order of pedestrian departure. For each GLM, we ran multi-model inferences to rank candidate models according to (i) their respective Akaike information criterion after correction for small sample sizes (AICc) and (ii) normalized Akaike weights (AICw) (Burnham and Anderson, 2004). $\triangle \mathrm{AICc}$ is the difference in AICc between a given model and the model with the lowest AICc. AICw indicates the probability that a given model will be the best among candidate models (Burnham and Anderson, 2004; Burnham et al., 2011; Johnson and Omland, 2004).

All models were considered, from the null model ( 0 variable) to the full models (all tested variables). Models were ranked and compared according to their AICc, with the lowest AICc indicating the best model. Models with a difference of AICc ( $\triangle \mathrm{AICc})<4$ were considered equally possible candidates and their statistics (estimate, z-score and p-value) were averaged according to Barton's method (2013). The null model was also included as a possible candidate but was never among the models with lowest AICc. Averaged model 
coefficients were obtained for models with a $\triangle \mathrm{AICc}<4$. Model inference and averaging were carried out with the R package 'MuMIn’ (Bartoń, 2013).

A Pearson correlation test was used to assess whether the relative number of abandons and accelerations increased or decreased significantly according to the pedestrian's departure order (this analysis was carried out until the sixth rank, as no pedestrians were observed hesitating after this rank order).

Statistical analyses were performed using Rstudio 1.0.143 (Team RStudio, 2015) and the significance level was set at 0.05 .

\section{RESULTS}

\section{a) Factors affecting uncertainty behaviour}

We observed 80 uncertainty behaviours for 1,464 infractions at Strasbourg (i.e. $5.46 \%$ ) whilst 38 uncertainty behaviours were observed for 369 infractions at Nagoya $(10.29 \%)$, which is different $\left(\mathrm{chi}^{2}=11.4, \mathrm{p}<0.001\right)$. The gender of the pedestrian had no effect on the likelihood of hesitating while crossing against a red light in either country $\left(\operatorname{chi}^{2}<0.48\right.$, $\mathrm{p}>0.490$ ). For the age variable, a zig-zag product on the likelihood of hesitating was observed on the graph for Japanese pedestrians. This is probably due to the small dataset with uncertainty behaviours. The age graph for French pedestrians seemed more coherent, with the highest number of uncertainty behaviours recorded for 20-year-old pedestrians and the lowest for their 60-year-old counterparts (Figure 2). The tendency to hesitate (K-s test, $\mathrm{W}=6074$, pvalue $=0.06178$ ) was observed in the first pedestrians to cross the road in France (average $\pm \operatorname{stdv}=1.8 \pm 1.2$ ), whilst the same tendency was shown by pedestrians following individuals who had already started crossing in Japan (average $\pm \operatorname{stdv}=2.2 \pm 1.6$ ). We observed ten hesitating individuals using their phones ( $8.4 \%$ of hesitating individuals), which is no higher than the rate of individuals using their phones whilst crossing at a red pedestrian light $(8.6 \%)$.

The uncertainty time GLM showed that this dependent variable is influenced by the country (Table 2, $\mathrm{z}=2.134, \mathrm{p}=0.033)$, by being accompanied $(\mathrm{z}=2.526, \mathrm{p}=0.012)$ and by the [country*accompanied] interaction $(\mathrm{z}=2.087, \mathrm{p}=0.037)$. Uncertainty time appears to be longer in Japan, and longer when the pedestrian is alone in both countries, but more importantly this last effect is enhanced in Japan (i.e. a stronger effect was observed in Japan, with longer uncertainty times when alone). Other factors (see Table 2) did not affect the time of uncertainty. 
After the 118 scored uncertainty behaviours, pedestrians showed 90 abandons (76.3\%) and 28 accelerations (23.7\%). Abandoning a crossing therefore appears then more probable than accelerating. Surprisingly, the percentage of accelerations is higher in Japan compared to France (34.2\% and $18.7 \%$, respectively). The GLM for the types of subsequent behaviours (abandoning or accelerating, Table 3) showed that only the number of lanes [2x1] is significant $(\mathrm{z}=3.445, \mathrm{p}=0.001)$ : the abandon rate increased with the number of lanes, whilst the acceleration rate decreased (Figure 3). Due to our small sample size, we did not obtain a significant effect for [2x2] and [2x3] lanes (where only abandon behaviours were observed). Concerning the departure order $(\mathrm{z}=1.682, \mathrm{p}=0.093)$, a Spearman correlation test on the percentage of abandons/accelerations according to the pedestrian departure order revealed that the relative number of abandons decreases with the departure order, whilst the relative number of accelerations increases ( rho $_{a b a n d o n}=-0.86$, rho $_{\text {acceleration }}=0.86, p=0.026$, Figure 4 ).

\section{DISCUSSION}

This study highlights the factors influencing the uncertainty behaviour of pedestrians crossing against the red light and the subsequent behaviours, namely abandoning or accelerating the crossing. Japanese citizens are known to respect social rules more than French or even western citizens (Benedict, 2005; Komiya, 1999; Markus and Kitayama, 1991; Ito, 1989). These cultural differences are not explained by differences in environmental factors but rather by the emergence of social conventions (Centolla and Baronchelli, 2015). A previous study showed that only $2.1 \%$ of crossings at signalized intersections are illegal in Japan, compared to $41.9 \%$ in France (Pelé et al., 2017). However, only 5\% of French pedestrians showed uncertainty behaviours when crossing against the red light, compared to $10 \%$ of the Japanese pedestrians. It is important to note that it was impossible to carry out observations of a road with $3 * 2$ lanes in Strasbourg to compare with our observations in Nagoya. This limitation could decrease the ratio of illegal crossings and the ratio of uncertainty behaviours in Japan compared to France.

The uncertainty time is also longer in Nagoya (Japan, $1.55 \pm 1.45 \mathrm{~s}$ ) than in Strasbourg (France, 1.22 $\pm 0.97 \mathrm{~s}$ ). These two elements -higher rate and longer time in Japan - lead us to suggest that Japanese pedestrians who crossed illegally mostly did so by following other pedestrians. Indeed, hesitating seems to occur in Japan when a pedestrian follows others already crossing against the red light (rather than due to inattention from using their phone, see explanations below) then realises the misevaluation. Pelé et al. (2017) have already 
described this phenomenon in Japanese pedestrians. Indeed, pedestrians show a strong mimetism by following social information when they cross the road, sometimes inadvertently crossing against the red light (Faria, 2010; Pelé et al., 2017). This could explain why surprised pedestrians hesitate when they realise that they are crossing illegally. The information they obtain after starting to cross might be personal (by checking the light colour or seeing a car arriving) or social (noticing that other pedestrians do not cross). These conclusions may be confirmed by the fact that accompanied pedestrians in Japan showed a lower uncertainty time than pedestrians who crossed alone: the accompanied pedestrian could be therefore helped by social information. These results and previous findings (Pelé et al., 2017) confirm a strong inter-country difference in the causes of illegal road-crossings and the causes of uncertainty behaviour. Pelé et al. (2017) showed that whilst most pedestrians deliberately cross against the red light in France, they appear to do so accidentally in Japan.

Many studies have shown an age and gender effect on crossing behaviours (Tom and Granié, 2011; Holland and Hill, 2007; Oxley et al., 1997). However, no such effects were observed in our study for the uncertainty behaviour rate, or for the uncertainty time and its possible subsequent behaviours. We suggest that this absence of effect may be due to two consecutive indirect filters resulting from our hesitating pedestrian population and linked to the data set: we studied uncertainty behaviours in a population of individuals who showed such behaviours (compared to those who did not show them). In turn, this population was part of a larger population of individuals: those crossing at a red light (compared to those who crossed at the green light). We assume that these two consecutive filters resulting from our dataset could lead us to solely observe pedestrians presenting a certain level of risk taking. For instance, women and/or elderly pedestrians, known to take fewer risks than men or adolescents (Sueur et al., 2013; Faralla et al., 2013; Wilson and Dally, 1985) did not cross at the red light or rarely did so, thus could bias the dataset and providing non-significant results for age and/or sex effect on uncertainty. We also expected men to display more accelerations than women, but this gender effect does not appear to be statistically significant, again probably due to the aforementioned filtering. We checked this filtering hypothesis in our dataset: this was checked with Japan pedestrians but not with French ones. Indeed, we observed a change in gender-ratio between total crossings and illegal crossings and between illegal crossings and uncertainty behaviours in Japan but this was not the case with France (see Table 4). Considering a possible age effect, we expected more uncertainty behaviours from the youngest (10-year-old) and oldest (more than 60-year-old) pedestrians than in other age categories. However, the results were inconsistent in Japan (Figure 2a) and were 
unexpected in France (Figure 2b), with 20-year-old French pedestrians showing the highest rate of uncertainty behaviours and 60-year-old pedestrians displaying the lowest rate of uncertainty behaviours. This result may be explained by our low sample size, but we cannot exclude the possible effect of decreasing cognitive abilities with age (Brown and Ott, 2004; Farias et al., 2005; Iosa et al., 2014; Salthouse, 1996), responsible for a lack of reaction or absence of uncertainty behaviour in the elderly when in risky situations (Holland and Hill, 2007; Oxley at al., 1997).

Whilst we did not observe an effect of environmental variables on the uncertainty time, we did observe an effect on the probability of an acceleration or abandon behaviour after uncertainty. A higher number of lanes resulted in a decrease in the probability that a pedestrian would accelerate and an increase in the probability that they would abandon the crossing. Only abandon scenarios were displayed by pedestrians illegally crossing roads with six lanes (three lanes in each direction). As the number of lanes increases, the number of vehicles and subsequently the risk of accidents increases. Thereby, abandoning the crossing appears to be a safer behaviour for pedestrians than accelerating. Indeed, we decrease our perception abilities when rushing, and we thus increase the probability of an error of judgement. This processing-speed theory (Salthouse, 1996) - where accuracy of decisions decreases with speed (Pelé and Sueur, 2013; Nagengast et al., 2011; Sueur and Pelé, 2015) has been well demonstrated for driving speed but can also be confirmed in pedestrians (Aarts and Schagen, 2006; Kloeden et al., 2002; Kloeden et al., 2001).

Our results also show that the probability of abandoning decreases with the departure order of the pedestrian (i.e. with the number of pedestrians who have already crossed or started crossing) while the probability to accelerate increases. This mimetic effect is due to conformity bias (Cialdini and Goldstein, 2004), and increases the risk of being injured or killed. Referred to as "sheep" or "herd" behaviour, this copying behaviour is well known in human beings in domains such as financial markets, fashion, purchasing or crowd behaviour (Banerjee, 1992; Bikhchandani et al., 1992; Chen, 2008; Hirshleifer et al., 2003; Mawson, 2005; Raafat et al., 2009). It seems to be deeply rooted in human behaviour due to the gregariousness and sociality of the human species, and can be observed in many other social animal species (Couzin and Krause, 2003; King and Sueur, 2011; Krause and Ruxton, 2002). However, it may also reflect a sense of safety in numbers; car drivers are more likely to see a group and are hence more likely to slow down or stop, even if they have right of way (Harrell, 1991; Rosenbloom, 2009). 
Finally, contrary to what we expected and what has been described in the literature

410 (McKnight and McKnight, 1993; Neider et al., 2010; Strayer et al., 2003), we did not observe 411 a decrease in attention when pedestrians were using a phone. Only ten hesitating individuals 412 were observed using their phone ( $8.4 \%$ of the sample size). This does not seem no higher than 413 the rate of individuals using a phone whilst crossing against the red light (8.6\%), and these 414 pedestrians did not show a higher uncertainty time than other pedestrians. The rate recorded 415 in our study is lower than the $20 \%$ of distracted pedestrians found by Bungum and colleagues 416 (2005). Again, this result may be due to the low sample size caused by the double filtering 417 (see above).

418 Overall, combined with the findings of previous studies (Pelé et al., 2017), these results indicate the existence of two categories of rule breakers among pedestrians, namely those who deliberately do so (as described in Pelé et al. 2017) and those who do so inadvertently (shown here through uncertainty behaviour). This second category appears to represent the most vulnerable pedestrians, because they seem to misevaluate a safety crossing. Additional signals could be set up to provide more adequate protection of pedestrians at accident-prone intersections. Sound signals have already been set up to help impaired or blind people (Tauchi et al., 2000; Tauchi et al., 1998). In our case, we could imagine less pleasant signals such as the new water spray systems that surprised rule-breaking pedestrians in China's Hubei Province (BBC News). Further research will then be needed to measure the success rate of such innovative systems in saving lives.

\section{ACKNOWLEDGEMENTS}

We thank Caroline Bellut, Elise Debergue, Charlotte Gauvin, Anne Jeanneret, Thibault Leclere, Lucie Nicolas, Florence Pontier and Diorne Zausa for their help collecting data, and Cédric Sueur for his help with the analyses. We are grateful to Kunio Watanabe and Hanya Goro (Primate Research Institute, Kyoto University) for their help in authorizing data collection and to Joanna Munro for English language editing. We also thank the three anonymous referees for their constructive comments, which helped to improve this paper. 


\section{BIBLIOGRAPHY}

Aarts, L., \& Van Schagen, I., 2006. Driving speed and the risk of road crashes: A review. Accident Analysis and Prevention, 38(2), 215-224.

Altmann, J., 1974. Observational Study of Behavior: Sampling Methods. Behaviour, 49(3/4), 227-267.

Banerjee, A. V., 1992. A Simple Model of Herd Behavior. The Quaterly Journal of Economics. 107(3), 797-817.

Bartoń, K., 2013. MuMIn: multi-model inference. $R$ package version. 1(5).

BBC News. If you jaywalk in China - prepare to get a soaking. https://www.bbc.com/news/av/world-asia-china-43836365/china-deters-jaywalkerswith-water-spray-at-crossing. Published April 20, 2018. Accessed June 11, 2018.

Benedict, R., 2005. The Chrysanthemum and the Sword: Patterns of Japanese Culture, Boston, Houghton Mifflin Harcourt.

Bode, N. W. F., Wagoum, A. U. K., \& Codling, E. A., 2015. Information use by humans during dynamic route choice in virtual crowd evacuations. Royal Society Open Science, 2(1), 140410.

Bikhchandani, S., Hirshleifer, D., \& Welch, I., 1992. A Theory of Fads, Fashion, Custom, and Cultural Change as Informational Cascades. Journal of Political Economy, 100(5), 9921026.

Boos, M., Pritz, J., Lange, S., \& Belz, M., 2014. Leadership in Moving Human Groups. PLOS Computational Biology, 10(4), e1003541.

Brown, L. B., \& Ott, B. R., 2004. Driving and dementia: a review of the literature. Journal of geriatric Psychiatry and Neurology, 17(4), 232-240.

Bunn, F., Collier, T., Frost, C., Ker, K., Roberts, I., \& Wentz, R., 2003. Traffic calming for the prevention of road traffic injuries: systematic review and meta-analysis. Injury Prevention, 9(3), 200-204.

Bungum, T. J., Day, C., \& Henry, L. J., 2005. The association of distraction and caution displayed by pedestrians at a lighted crosswalk. Journal of Community Health, 30(4), 269-279.

Burnham, K. P., \& Anderson, D. R., 2004. Multimodel inference understanding AIC and BIC in model selection. Sociological Methods \& Research, 33(2), 261-304.

Burnham, K. P., Anderson, D. R., \& Huyvaert, K. P., 2011. AIC model selection and multimodel inference in behavioral ecology: some background, observations, and comparisons. Behavioral Ecology and Sociobiology, 65(1), 23-35. 
Centola, D., \& Baronchelli, A., 2005. The spontaneous emergence of conventions: An experimental study of cultural evolution. PNAS, 201418838.

Chen, Y-F., 2008. Herd behavior in purchasing books online. Computers in Human Behavior, 24(5), 1977-1992.

Cialdini, R. B., \& Goldstein, N. J., 2004. Social influence: Compliance and conformity. Annual Review of Psychology, 55, 591-621.

Couzin, I. D., \& Krause, J., 2003. Self-organization and collective behavior in vertebrates. Advances in the Study of Behavior, 32, 1-75.

Desapriya, E., Subzwari, S., Sasges, D., Basic, A., Alidina, A., Turcotte, K., \& Pike, I., 2010. Do light truck vehicles (LTV) impose greater risk of pedestrian injury than passenger cars? A meta-analysis and systematic review. Traffic Injury Prevention, 11(1), 48-56.

Dyer, J. R., Johansson, A., Helbing, D., Couzin, I. D., \& Krause, J., 2008. Leadership, consensus decision making and collective behaviour in humans. Philosophical Transactions of the Royal Society B: Biological Sciences, 364(1518), 781-789.

Faralla, V., Innocenti, A., \& Venturini, E., 2013. Risk Taking and Social Exposure. LABSI Working Papers, 46.

Faria, J. J., Krause, S., \& Krause, J., 2010. Collective behavior in road crossing pedestrians: the role of social information. Behavioral Ecology, 21(6), 1236-1242.

Farias, S. T., Mungas, D., \& Jagust, W., 2005. Degree of discrepancy between self and other-reported everyday functioning by cognitive status: Dementia, mild cognitive impairment, and healthy elders. International Journal of Geriatric Psychiatry, 20(9), 827-834.

Friard, O., \& Gamba, M. (2016). BORIS: a free, versatile open-source event-logging software for video/audio coding and live observations. Methods in Ecology and Evolution, 7(11), 1325-1330.

Hamed, M. M., 2001. Analysis of pedestrians' behavior at pedestrian crossings. Safety Science, 38(1), 63-82.

Harrell, W. A., 1991. Factors influencing pedestrian cautiousness in crossing streets. The Journal of Social Psychology, 131(3), 367-372.

Hingson, R., \& Winter, M., 2003. Epidemiology and consequences of drinking and driving. Alcohol Research and Health, 27(1), 63-78.

Hirshleifer D., \& Hong Teoh, S., 2003. Herd Behaviour and Cascading in Capital Markets: a Review and Synthesis. European Financial Management, 9(1), 25-66. 
Holland, C., \& Hill, R., 2007. The effect of age, gender and driver status on pedestrians' intentions to cross the road in risky situations. Accident Analysis \& Prevention, 39(2), 224-237.

Iosa, M., Fusco, A., Morone, G., \& Paolucci, S., 2014. Development and decline of upright gait stability. Frontiers in Aging Neuroscience, 6, 14.

Ito, Y., 1989. Socio-cultural backgrounds of Japanese interpersonal communication style. Civilisations, 39, 101-128.

Johnson, J. B., \& Omland, K. S., 2004. Model selection in ecology and evolution. Trends in Ecology \& Evolution, 19(2), 101-108.

King, A. J., \& Sueur, C., 2011. Where Next? Group Coordination and Collective Decision Making by Primates. International Journal of Primatology, 32(6), 1245-1267.

Kloeden, C. N., McLean, J., \& Glonek, G. F. V., 2002. Reanalysis of Travelling Speed and the Risk of Crash Involvement in Adelaide South Australia. Australian Transport Safety Bureau.

Kloeden, C. N., Ponte, G., \& McLean, J., 2001. Travelling Speed and Risk of Crash Involvement on Rural Roads. Australian Transport Safety Bureau.

Komiya, N., 1999. A cultural study of the low crime rate in Japan. British Journal of Criminology, 39(3), 369-390.

Krause, J., \& Ruxton, G. D., 2002. Living in Groups. Oxford, Oxford University Press.

Markus, H., \& Kitayama, S., 1991. Culture and the Self - Implications for Cognition, Emotion, and Motivation. Psychological Review, 98(2), 224-253.

Mawson, A. R., 2005. Understanding Mass Panic and Other Collective Responses to Threat and Disaster. Psychiatry: Interpersonal and Biological Processes, 68(2), 95-113.

Nagengast, A. J., Braun, D. A., \& Wolpert, D. M., 2011. Risk sensitivity in a motor task with speed-accuracy trade-off. Journal of Neurophysiology, 105(6), 2668-2674.

Neider, M. B., McCarley, J. S., Crowell, J. A., Kaczmarski, H., \& Kramer, A. F., 2010. Pedestrians, vehicles, and cell phones. Accident Analysis and Prevention, 42(2), 589594.

Osman, L. M., 1982. Conformity or compliance? A study of sex differences in pedestrian behaviour. British Journal of Social Psychology, 21(1), 19-21.

Oxley, J., Fildes, B., Ihsen, E., Charlton, J., \& Day, R., 1997. Differences in traffic judgements between young and old adult pedestrians. Accident Analysis and Prevention, 29(6), 839-847. 
Pelé, M., \& Sueur, C., 2013. Decision-making theories: linking the disparate research areas of individual and collective cognition. Animal Cognition, 16(4), 543-556.

Pelé, M., Bellut, C., Debergue, E., et al., 2017. Cultural influence of social information use in pedestrian road-crossing behaviours. Royal Society Open Science, 4(2), 160739.

Pelé, M., Deneubourg, J. L., \& Sueur, C., (2019) Decision-making processes underlying pedestrian behaviours at signalised crossings: Part 2. Do pedestrians show cultural herding behaviour? Safety, 5(4): 82.

Porter, A., 1998. Reducing road traffic. First fatal car crash in Britain occurred in 1898. BMJ, 317(7152), 212.

Raafat, R. M., Chater, N., \& Frith, C., 2009. Herding in humans. Trends in Cognitive Sciences, 13(10), 420-428.

Rosen, E., Stigson, H., \& Sander, U. 2011. Literature review of pedestrian fatality risk as a function of car impact speed. Accident Analysis and Prevention, 43(1), 25-33.

Rosenbloom, T., 2009. Crossing at a red light: Behaviour of individuals and groups. Transportation Research Part F: Traffic Psychology and Behaviour, 12(5), 389-394.

Salthouse, T. A., 1996. The processing-speed theory of adult age differences in cognition. Psychological Review, 103(3), 403.

Sueur, C., \& Pelé, M., 2015. Risk should be objectively defined: reply to Zentall and Smith. Animal Cognition, 18(4), 981-983.

Sueur, C., Class, B., Hamm, C., Meyer, X., \& Pelé, M., 2013. Different risk thresholds in pedestrian road crossing behaviour: a comparison of French and Japanese approaches. Accident Analysis and Prevention, 58, 59-63.

Tapiro, H., Borowsky, A., Oron-Gilad, T., \& Parmet, Y., 2016a, Where do older pedestrians glance before deciding to cross a simulated two-lane road? A pedestrian simulator paradigm. Proceedings of the Human Factors and Ergonomics Society Annual Meeting, Washington DC, United-States, September 2016, 60(1), 11-15.

Tapiro, H;, Oron-Gilad, T; Parmet, Y., 2016b. Cell Phone conversation and child pedestrian's crossing behavior: Simulation study. Safety Science, 89, 36-44.

Tapiro, H., Oron-Gilad, T., Parmet, Y., 2018. The Effect of Environmental Distractions on Child Pedestrian's Crossing Behavior. Safety Science, 106, 249-229.

Tauchi, M., Sawai, H., Takato, J., Yoshiura, T., Takahara, S., \& Suzuki, T., 2000. Audible traffic signal for visually impaired persons using multiple sound outputs. US Patent and Trademark Office, 6(127), 943. 
Tauchi, M., Yoshiura, T., Sawai, H., Takato, J., \& Takeuchi, K., 1998. A new audible traffic signal that helps blind pedestrians to cross intersections by providing navigation clues. Proceedings of the $5^{\text {th }}$ World Congress on Intelligent Transport Systems, Seoul, SouthKorea, October 1998, 3050, 1-8.

Team Rs., 2015. RStudio: integrated development for R. RStudio, Inc, Boston, MA URL http://www rstudio com. 2015

Tom, A., \& Granié, M-A., 2011. Gender differences in pedestrian rule compliance and visual search at signalized and unsignalized crossroads, Accident Analysis and Prevention, 43(5), 1794-1801.

Wilson, M., \& Daly, M., 1985. Competitiveness, risk taking, and violence: The young male syndrome. Ethology and Sociobiology, 6(1), 59-73.

Yannis, G., Papadimitriou, E., \& Theofilatos, A., 2013. Pedestrian gap acceptance for midblock street crossing. Transportation Planning and Technology, 36(5), 450-462.

Zhuang, X., \& Wu, C., 2011. Pedestrians' crossing behaviors and safety at unmarked roadway in China. Accident Analysis \& Prevention, 43(6), 1927-1936. 
Table 1: Details concerning the studied sites in Strasbourg (France) and Nagoya (Japan). A total of 80 and 38 uncertainty behaviours were observed in France and in Japan, respectively, on the lines "Number of uncertainty behaviours counted during 6 hours of observation". Pictures of the different intersections are available in the Supplementary Material section of

Pelé et al. 2017, via this link: https://doi.org/10.6084/m9.figshare.c.3677176.v1

\begin{tabular}{|c|c|c|c|c|}
\hline \multicolumn{5}{|c|}{ Strasbourg-France } \\
\hline Sites & Train Station & Pont des Corbeaux & Place Broglie & \\
\hline Coordinates & $\begin{array}{c}48.584474 \\
7.736135\end{array}$ & $\begin{array}{c}48.579509 \\
7.750745\end{array}$ & $\begin{array}{c}48.584559, \\
7.748628\end{array}$ & \\
\hline Number of lanes in each direction & 1 & 2 & 1 & \\
\hline Mean pedestrian flow per hour & 667 & 612 & 850 & \\
\hline Mean road-crossing speed $\left({\mathrm{m} . \mathrm{s}^{-}}^{1}\right)$ & $0.96 \pm 0.05$ & $1.11 \pm 0.29$ & $1.01 \pm 0.16$ & \\
\hline Data collection dates & 02/07-07/07/2014 & $01 / 10-25 / 10 / 2014$ & $15 / 02-09 / 03 / 2015$ & \\
\hline $\begin{array}{c}\text { Number of uncertainty behaviours } \\
\text { counted during } 6 \text { hours of } \\
\text { observation }\end{array}$ & 36 & 23 & 21 & \\
\hline Percentage of illegal crossings & $58.29 \%$ & $32.92 \%$ & $44.02 \%$ & \\
\hline \multicolumn{5}{|c|}{ Nagoya-Japan } \\
\hline Sites & Train Station & Maruei & Excelco & Osu-Kannon \\
\hline Coordinates & $\begin{array}{l}35.170824, \\
136.884328 \\
\end{array}$ & $\begin{array}{l}35.168638 \\
136.905740 \\
\end{array}$ & $\begin{array}{l}35.166891, \\
136.907284\end{array}$ & $\begin{array}{l}35.159316, \\
136.901697\end{array}$ \\
\hline Number of lanes in each direction & 3 & one-way street & 1 & 1 \\
\hline Mean pedestrian flow per hour & 480 & 645 & 869 & 814 \\
\hline Mean road-crossing speed $\left({\mathrm{m} . \mathrm{s}^{-}}^{-1}\right)$ & $1.10 \pm 0.22$ & $1.15 \pm 0.21$ & $0.98 \pm 0.21$ & $1.07 \pm 0.18$ \\
\hline Data collection dates & $13 / 06-05 / 07 / 2011$ & \multicolumn{3}{|c|}{$27 / 01-05 / 02 / 2015$} \\
\hline $\begin{array}{c}\text { Number of uncertainty behaviours } \\
\text { counted during } 6 \text { hours of } \\
\text { observation }\end{array}$ & 3 & 15 & 4 & 16 \\
\hline Percentage of illegal crossing & $8.34 \%$ & $14.53 \%$ & $2.11 \%$ & $8.86 \%$ \\
\hline
\end{tabular}



chosen is $[1 \times 1]$.

\begin{tabular}{|l|c|c|c|c|c|}
\hline \multicolumn{1}{|c|}{ Independent variables } & Estimate & Std. Error & Adjusted & SE value & $\operatorname{Pr}(>|\mathbf{z}|)$ \\
\hline (Intercept) & 0.869 & 0.281 & 0.284 & 3.064 & 0.002 \\
\hline Accompanied [Yes] & -0.733 & 0.288 & 0.290 & 2.526 & 0.012 \\
\hline Country [France] & -0.629 & 0.292 & 0.295 & 2.134 & 0.033 \\
\hline Accompanied [Yes]* Country [France] & 0.660 & 0.313 & 0.316 & 2.087 & 0.037 \\
\hline Number of waiting pedestrians & -0.014 & 0.014 & 0.014 & 1.033 & 0.302 \\
\hline Gender [Man] & -0.105 & 0.191 & 0.193 & 0.543 & 0.587 \\
\hline Number of lanes [2x1] & 0.368 & 0.286 & 0.289 & 1.275 & 0.202 \\
\hline Number of lanes [2x2] & 0.478 & 0.323 & 0.326 & 1.465 & 0.143 \\
\hline Number of lanes [2x3] & -0.224 & 0.487 & 0.493 & 0.455 & 0.649 \\
\hline Using a phone [Yes] & -0.157 & 0.260 & 0.263 & 0.595 & 0.552 \\
\hline Age & -0.003 & 0.005 & 0.005 & 0.547 & 0.584 \\
\hline Departure Order & -0.005 & 0.054 & 0.054 & 0.099 & 0.921 \\
\hline Gender [Man]*Country [France] & -0.200 & 0.303 & 0.306 & 0.652 & 0.514 \\
\hline
\end{tabular}

Table 2: Results of the model selection (see statistical analyses section) with the uncertainty time (in seconds) as a dependent variable. Considering the number of lanes, the reference

600 59

601 
603 Table 3: Results of the model selection (see statistical analyses section) with the type of 604 behaviour following uncertainty as dependent variable (abandon $=1$, acceleration $=0$ ).

\begin{tabular}{|l|c|c|c|c|c|}
\hline \multicolumn{1}{|c|}{ Independent variables } & Estimate & Std. Error & Adjusted SE & $\mathbf{z}$ value & $\operatorname{Pr}(>|\mathbf{z}|)$ \\
\hline (Intercept) & -0.574 & 1.067 & 1.075 & 0.534 & 0.593 \\
\hline Number of lanes [2x1] & 2.882 & 0.829 & 0.837 & 3.445 & 0.001 \\
\hline Number of lanes [2x2] & 0.625 & 0.874 & 0.883 & 0.708 & 0.479 \\
\hline Number of lanes [2x3] & 17.015 & 1377.357 & 1392.471 & 0.012 & 0.990 \\
\hline Departure Order & -0.303 & 0.178 & 0.180 & 1.682 & 0.093 \\
\hline Age & 0.023 & 0.018 & 0.018 & 1.273 & 0.203 \\
\hline Gender [Man] & 0.648 & 0.550 & 0.556 & 1.166 & 0.244 \\
\hline Number of waiting pedestrians & -0.054 & 0.050 & 0.051 & 1.072 & 0.284 \\
\hline Country [France] & 0.670 & 0.832 & 0.841 & 0.797 & 0.425 \\
\hline Using a phone [Yes] & 0.490 & 0.984 & 0.995 & 0.492 & 0.623 \\
\hline Accompanied [Yes] & -0.179 & 0.558 & 0.563 & 0.319 & 0.750 \\
\hline
\end{tabular}

605

606

607

608

609

610

611

612

613

614

615

616

617

618

619

620

621 
622 Table 4: Relative percentage of men and women in France and Japan according to the total 623 crossing (at red and green light), the illegal crossing (red light) and the uncertainty 624 behaviours.

\begin{tabular}{c|lccc}
\hline & & $\begin{array}{c}\text { Total } \\
\text { crossing }\end{array}$ & $\begin{array}{c}\text { Illegal } \\
\text { crossing }\end{array}$ & $\begin{array}{c}\text { Uncertainty } \\
\text { behaviours }\end{array}$ \\
\hline France & Men & 44.4 & 44.4 & 46.25 \\
& Women & 55.6 & 55.5 & 53.75 \\
& & & & \\
Japan & Men & 42.2 & 59 & 42.1 \\
& Women & 57.7 & 41 & 57.9 \\
\hline
\end{tabular}

625

626 
628

a. Abandon

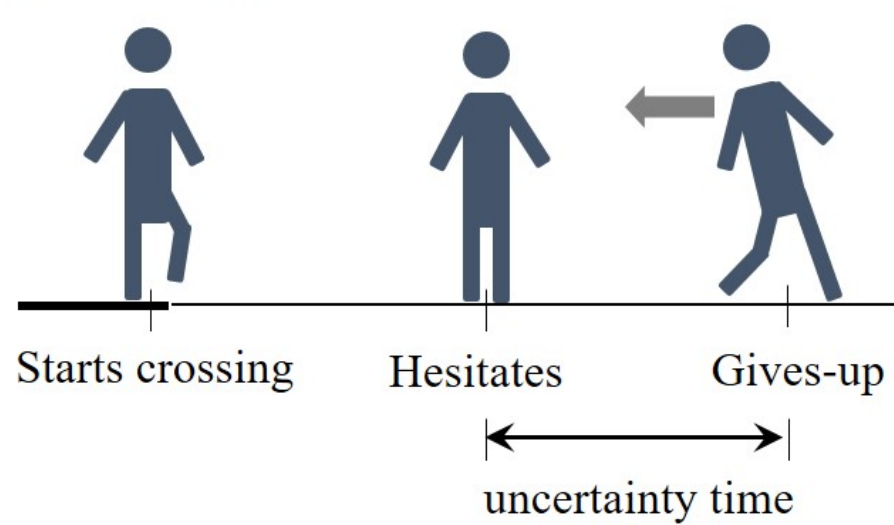

b. Acceleration
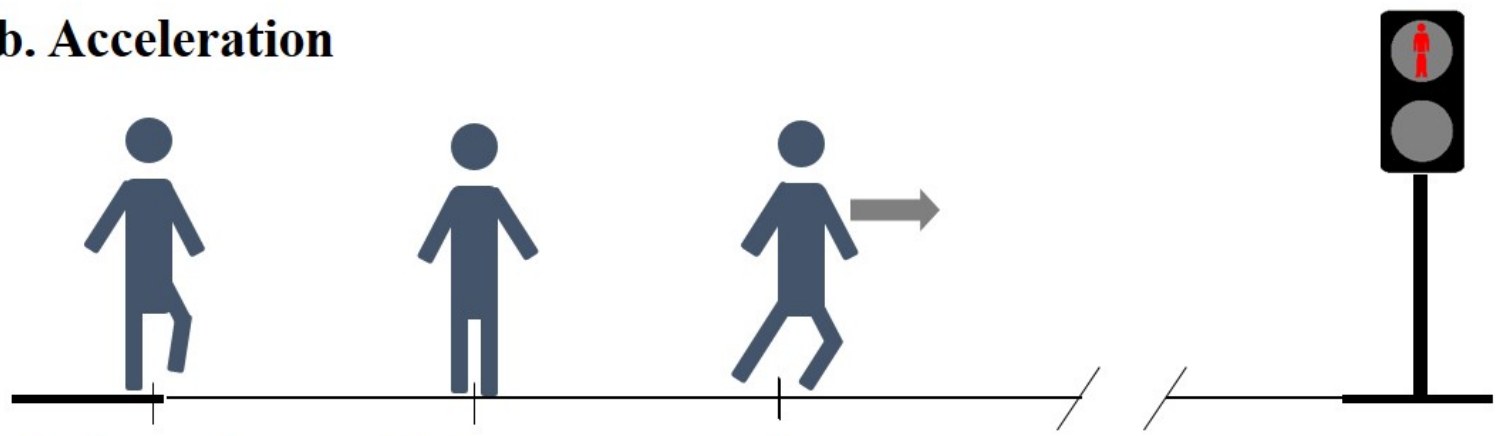

Starts crossing

Hesitates Accelerates

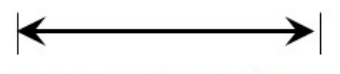

629

uncertainty time

630 Figure 1: Temporal scheme of the different behaviours scored in this study.

631

632

633

634

635

636

637

638

639

640

641

642 

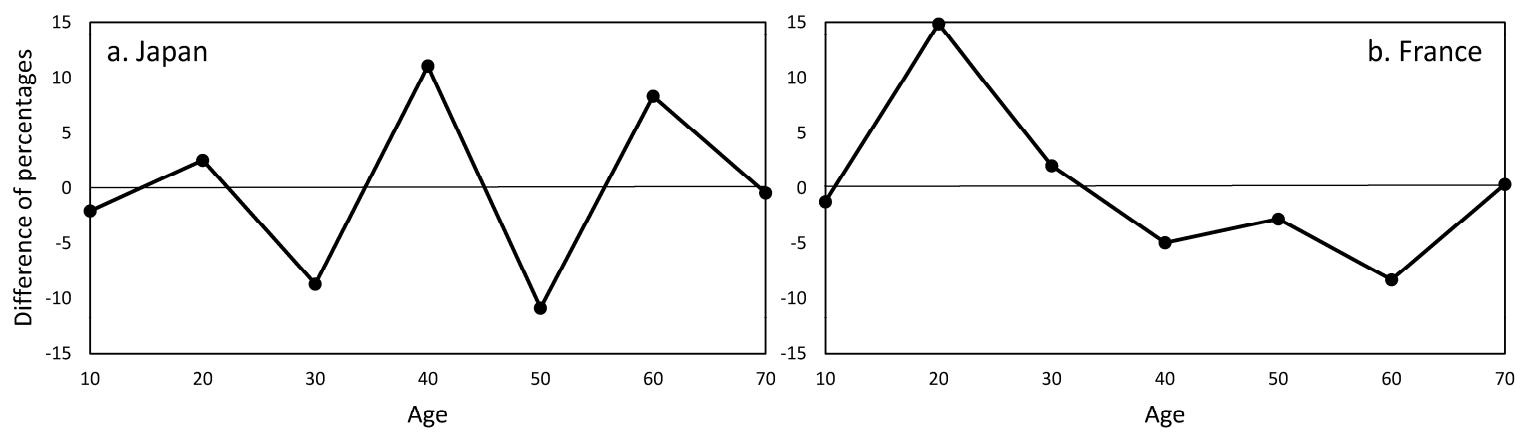

643

644 Figure 2: Differences in the percentages of 118 pedestrians showing uncertainty behaviours

$645(\mathrm{~N}=80$ for France and $\mathrm{N}=38$ for Japan) and 1,278 pedestrians of a sample size $(\mathrm{N}=551$ for 646 France and $\mathrm{N}=727$ for Japan) in a. Japan and b. France. Positive values showed that more 647 pedestrians in a given age category showed uncertainty behaviour than expected; negative 648 values showed that fewer pedestrians in a given age category showed uncertainty behaviour 649 than expected.

650

651

652

653

654

655

656

657

658

659

660

661

662

663

664

665

666

667

668

669

670 


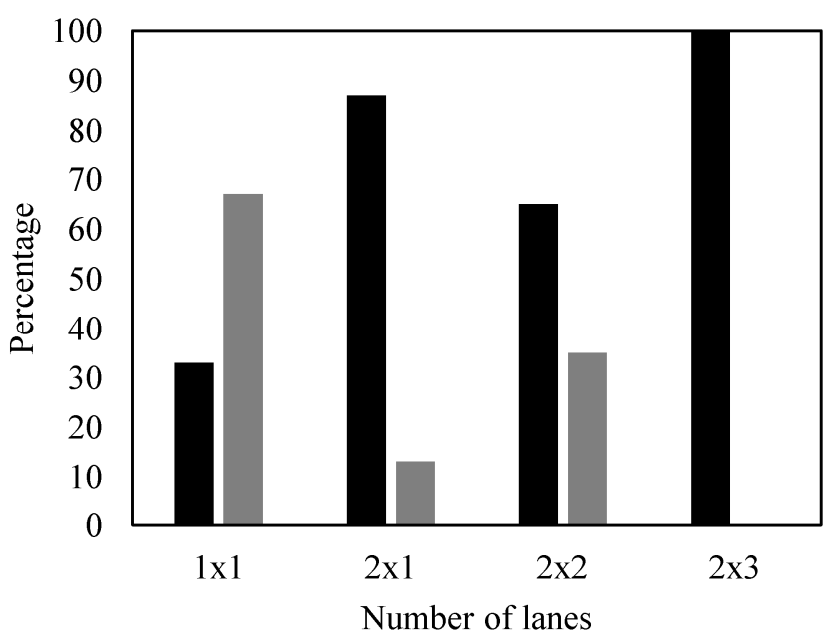

671

672 Figure 3: Percentage of abandons (black bars) and accelerations (grey bars) according to the 673 number of lanes.

674

675

676

677

678

679

680

681

682

683

684

685

686

687

688

689

690

691

692

693

694 


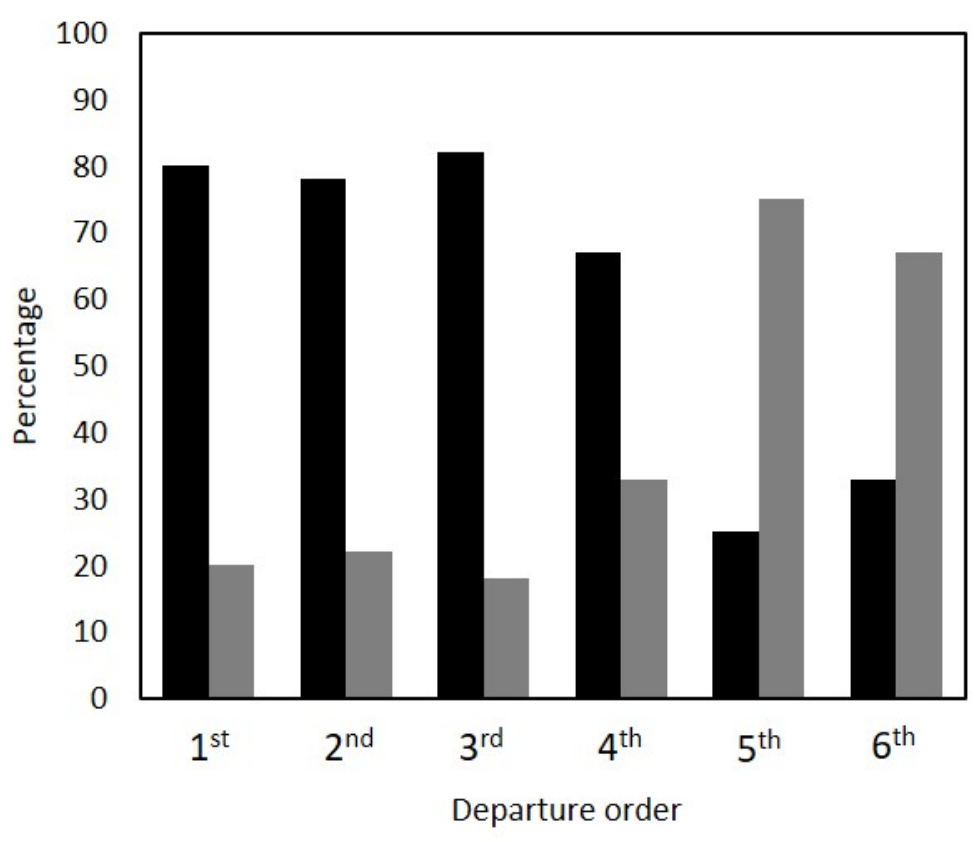

695

696 Figure 4: Rate of abandonment (black bars) and acceleration (grey bars) according to the 697 departure order of the pedestrian.

698

699

700

701

702 